\title{
Do disease specific characteristics add to the explanation of mobility limitations in patients with different chronic diseases? A study in the Netherlands
} Didi M W Kriegsman, Dorly J H Deeg, Jacques Th M van Eijk, Brenda W J H Penninx,
A Joan P Boeke
Institute for Research in Extramural Medicine

D M W Kriegsman

J Th $M$ van Eijk

B W J H Penninx

A J P Boeke

\section{Department of}

General Practice, Nursing Home

Medicine and Social

Medicine

D M W Kriegsman

$\mathrm{J}$ Th $M$ van Eijk

A J P Boeke

Department of Psychiatry and Department of Sociology and Social Gerontology

D J H Deeg

Vrije Universiteit, Amsterdam, the Netherlands

Correspondence to: Dr D M W Kriegsman, EMGO Institute, Van der Boechorststraat 7, $1081 \mathrm{BT}$ Amsterdam, the Netherlands.

\begin{abstract}
Study objectives-To determine whether disease specific characteristics, reflecting clinical disease severity, add to the explanation of mobility limitations in patients with specific chronic diseases.

Design and setting-Cross sectional study of survey data from community dwelling elderly people, aged 55-85 years, in the Netherlands.

Participants and methods-The additional explanation of mobility limitations by disease specific characteristics was examined by logistic regression analyses on data from 2830 community dwelling elderly people.

Main results-In the total sample, chronic non-specific lung disease, cardiac disease, peripheral atherosclerosis, diabetes mellitus, stroke, arthritis and cancer (the index diseases), were all independently associated with mobility limitations. Adjusted for age, sex, comorbidity, and medical treatment disease specific characteristics that explain the association between disease and mobility mostly reflect decreased endurance capacity (shortness of breath and disturbed night rest in chronic nonspecific lung disease, angina pectoris and congestive heart failure in cardiac disease), or are directly related to mobility function (stiffness and lower body complaints in arthritis). For atherosclerosis and diabetes mellitus, disease specific characteristics did not add to the explanation of mobility limitations.

Conclusions-The results provide evidence that, to obtain more detailed information about the differential impact of chronic diseases on mobility, disease specific characteristics are important to take into account.
\end{abstract}

(F Epidemiol Community Health 1997;51:676-685)

The role of chronic diseases as determinants of mobility limitations is intuitively important, but not well defined. A higher number of chronic diseases is consistently associated with a higher prevalence of mobility limitations, ${ }^{1-3}$ and longitudinally with a higher incidence of mobility loss. ${ }^{4}$ However, these associations do not provide information on the influence of different specific chronic diseases. Recently, more studies have been focused on associations between specific chronic diseases and mobility. ${ }^{4-9}$ In elderly people, the specific chronic diseases that are most consistently associated with either a higher prevalence or higher incidence of mobility limitations include arthritis, ${ }^{26-9}$ cardiac diseases, ${ }^{24}{ }^{7-9}$ cerebrovascular disorders, ${ }^{246-8}$ chronic obstructive pulmonary disease, ${ }^{7-9}$ diabetes mellitus ${ }^{2489}$ and, to a lesser extent, cancer ${ }^{28}{ }^{8}$ and atherosclerosis. ${ }^{2}$

It may, however, be doubted whether currently used measurements of chronic diseases are sufficiently detailed to obtain accurate information on the associations between specific chronic diseases and mobility. The meas urement of chronic diseases in population surveys is mostly confined to self reports pertaining to the presence or absence of a specific disease. The accuracy of patients' self reports regarding the presence of chronic diseases is generally considered adequate although this was shown to be different for specific chronic conditions. ${ }^{10-13}$ It has been stated that the clinical severity of the disease should be taken into account in studies on the associations between specific chronic diseases and mobility. ${ }^{4}$

Methods that are used by physicians in clinical practice can be of value in the assessment of clinical severity of chronic diseases. Physicians implicitly judge the clinical severity of a disease in their patients according to the presence of disease specific signs and symptoms. In acquiring the necessary knowledge, medical history questions are of primary importance. This is particularly the case for most chronic diseases. Surprisingly, the use of medical history questions, conditional on disease presence, as a method of improving the explanation of mobility limitations in survey studies has never been systematically evaluated. Information about the additional influence of these parameters of clinical severity is important for epidemiological research and health planning by providing a more detailed measure of chronic diseases that can be used to obtain a more precise picture of the burden of disease for both individuals and populations, and its association with outcome measures, such as mobility limitations. When the presence of certain disease specific symptoms, reflecting clinical severity, would seem to be strongly associated with mobility 
limitations, it may be possible to increase the accuracy of predictions for the future regarding the prevalence of mobility limitations, disability, and the use of health care facilities. In addition, intervention strategies directed at particularly those parameters of clinical severity of specific chronic diseases with the strongest associations with health outcomes may be developed to diminish the negative consequences of chronic diseases in the future.

In this study, we investigate whether the explanation of mobility limitations can be improved by adding questions directed at assessment of clinical severity, conditional on the self reported presence of a specific chronic disease. The influence of these parameters of clinical disease severity on the associations between chronic diseases and mobility limitations is examined for several chronic diseases that often afflict the elderly, and that have been repeatedly shown to influence physical functioning and thereby the ability of older people to live independently in the community. Seven specific chronic diseases with a high prevalence in the elderly population were selected: chronic non-specific lung disease (asthma, chronic bronchitis or pulmonary emphysema), cardiac disease (including myocardial infarction), peripheral atherosclerosis, cerebrovascular accident, diabetes mellitus, arthritis (rheumatoid arthritis or osteoarthritis) and malignant neoplasms.

The following questions will be considered:

(1) Does the self reported presence of specific chronic diseases explain self reported mobility limitations in community dwelling elderly people?

(2) Does the presence of signs and symptoms, reflecting clinical disease severity, add to the explanation of mobility limitations in patients with a specific chronic disease?

\section{Methods}

This study uses a cross sectional design. The data were collected as part of the first data collection cycle of the Longitudinal Aging Study Amsterdam (LASA), ${ }^{14}$ a 10 year longitudinal study on predictors and consequences of changes in physical, cognitive, emotional, and social functioning in older persons.

\section{HYPOTHESES}

For the first research question, we expected the association with mobility limitations to be most pronounced for those chronic diseases of which the clinical picture is characterised by symptoms of the locomotor system (stroke and arthritis), or by decreased endurance capacity (chronic non-specific lung disease, cardiac disease). The associations of the other specific chronic diseases (peripheral atherosclerosis, diabetes mellitus, and cancer) with mobility limitations are expected to be less pronounced.

The inclusion of disease specific parameters of clinical severity, as measured by medical history questions, is hypothesised to add to the explanation of mobility limitations especially in those chronic diseases that have the strongest association with mobility limitations. The symptoms that are expected to add most, are
KEY POINTS

- Self reports of chronic diseases are insufficient measures to explain mobility limitations in elderly people.

- Symptoms reflecting clinical disease severity add to the explanation of mobility limitations.

- Use of medical history questions is an adequate and cheap method to obtain information about the clinical severity of chronic diseases in survey studies.

- The accuracy of future predictions regarding prevalence of disability may be improved by using information from medical history questions.

those that most directly affect physical functioning (for example, symptoms of the lower body joints in patients with arthritis, and locomotor sequelae in stroke victims), or endurance capacity (for example, shortness of breath in patients with chronic non-specific lung disease, congestive heart failure in patients with cardiac disease). On the other hand, disease specific symptoms that are not directly associated with the locomotor system (for example, aphasia in stroke victims), or with endurance capacity (for example, having ever had wheezing breath, or a history of a recent exacerbation in patients with chronic non-specific lung disease, or a history of myocardial infarction in patients with cardiac disease) are not expected to add significantly to the explanation of mobility limitations.

\section{STUDY POPULATION}

A sample of people aged 55 to 85 years, stratified by age and sex according to expected attrition resulting from mortality at mid-term of LASA (after five years) in each age group, was drawn from the population registries of 11 municipalities in three culturally distinct geographical areas in the Netherlands. The cohort was recruited in 1991 for the NESTOR-LSN study "Living arrangements and social networks of older adults" (response rate $62.3 \%$ ). ${ }^{15}$ Although there was a decline in response with increasing age $(p<0.05)$, the stratification has ensured representativity on age, sex, and level of urbanisation of the sample. ${ }^{15}$ After 11 months, the participants in NESTOR-LSN were approached for the first LASA cycle. From the initial sample of 3805 persons who participated in NESTOR-LSN, a total of 3107 participated in the main interview of LASA $(81.7 \%)$. Data were collected in the period from September 1992 through October 1993. Details of the procedures and results of the field work are described elsewhere. ${ }^{16}$ Of the persons who did not participate, $260(6.8 \%$ of initial sample) proved to be ineligible (deceased or not able because of severe physical or cognitive disturbances, or both). Of the other 438 non-participants, $394(10.4 \%$ of initial sample) refused and 44 ( $1.2 \%$ of initial sample) could not be contacted. The corrected response percentage, excluding those who were ineligible after all, therefore was $87.6 \%$. Older 
age was significantly associated with refusal to participate $(p<0.001)$ and ineligibility because of severe physical or cognitive disturbances $(p<0.0001)$. Excluded for this study were participants who, at the time of the main interview, were institutionalised (living in a residential or nursing home or in a psychiatric hospital; $\mathrm{n}=126$ ), and those who were living independently but were unable to complete the full interview $(n=151)$, leaving a total number of 2830 ( $91.1 \%$ of all participants). As a result, the study population is a comparatively healthy selection of the original sample, although the stratified sampling frame guaranteed that sufficient numbers of subjects in the highest age group, as well as subjects with physical problems, were included.

\section{MEASUREMENTS}

\section{Determinants}

The presence of chronic diseases was assessed by asking the participants explicitly whether they had any of the following seven chronic diseases: chronic non-specific lung disease (asthma, chronic bronchitis or pulmonary emphysema), cardiac disease (including myocardial infarction), peripheral atherosclerosis, cerebrovascular accident, diabetes mellitus, arthritis (rheumatoid arthritis or osteoarthritis), and malignant neoplasms. If a disease was reported present, additional questions were asked concerning medical treatment (as a potential confounder) and disease specific symptoms and signs reflecting clinical severity of the disease. Pertinent medical history questions (questions that physicians use to get an impression of disease severity) were selected in close cooperation with general practitioners with expert knowledge on the specific chronic diseases. As far as possible, the items were derived from existing questionnaires such as the Rose Questionnaire for cardiac disease. ${ }^{17}$ For malignancies, a radiation oncologist was consulted. Table 1 presents the variables used in the analyses, as well as the absolute numbers of subjects in the "yes" category.

For cardiac diseases, the answers to the disease specific questions were used to distinguish separate diagnoses. The symptoms of diabetes mellitus pertained to the presence of diabetic complications: macrovascular complications (angina pectoris or intermittent claudication, or both), retinopathy, and peripheral neuropathy. For stroke, the presence of specific sequelae was included, namely locomotor disabilities, visual handicap, and expressive (such as difficulties in finding words) or perceptive (understanding written text) aphasia. For arthritis, both the presence of symptoms and the extent of joint involvement, as well as a history of joint surgery were taken into account. Because the numbers of respondents with a specific primary malignancy were too small to include them separately, malignancies were categorised according to overall five year survival rates after diagnosis. ${ }^{18}$ When the patient reported to have had two or more primary tumours, the category was based on the tumour with the worst prognosis.
The presence of other chronic diseases was $c$ assessed by asking respondents whether they had, at the moment of the interview, any other chronic disorder of at least three months' duration in addition to those that were explicitly asked. The category "other chronic diseases" includes, among others, neurological diseases, osteoporosis, neck and back problems, liver and kidney diseases, endocrinological diseases, varicose veins and venous insufficiency, hypertension, gastrointestinal diseases, allergies, consequences of accidents or surgery, chronic locomotor problems not covered elsewhere, dizziness, anaemia, disorders of the eyes or ears, and mental disorders.

\section{Outcome measure}

The presence of mobility limitations was assessed using three self report items pertaining to mobility activities in daily life: ability to walk up and down a 15 step staircase without stopping, use of private or public transportation, and cut own toenails. Answers on each item were originally coded as 0 "can be performed without difficulty", 1 "can be performed, but with difficulty", 2 "can only be performed with help" or 3 "cannot be performed at all". Based on the results of pilot studies, ${ }^{19}$ these items were shown to constitute the best scale out of a set of nine items. The selection of the three items was based on iterative reliability analysis, in which the items with the highest item rest correlation were retained without an important influence on the reliability coefficient. To determine whether the three mobility items, which cover a range of mobility related activities necessary to maintain autonomy in daily life, are equally important for the underlying dimension "mobility limitations" in subjects with specific chronic diseases, additional analyses were performed. Factor analyses were conducted for the three mobility items for each chronic disease separately, including only those subjects who reported to have only one of the specific chronic diseases. In this way, the relative importance of the separate items as part of the underlying dimension "mobility limitations" could be compared. The factor structure proved to be comparable between the specific chronic diseases, and was not different from that in subjects without any disease. Thus, the separate mobility items can be considered to represent a general underlying dimension for subjects with different diseases, and summing the three item scores results in a global index of mobility limitations, which is equally valid for each of the specific chronic diseases studied. Although the reliability of this three item mobility scale in the total study population was adequate (Cronbach's $\alpha 0.72$ ), and all items loaded on one factor (all factor loadings $>0.75$ ), use of a total score in linear regression analysis was precluded by an extremely skewed frequency distribution with $>60 \%$ having a total score of 0 (range $0-9$ ), and a non-random distribution of the residuals after multiple linear regression with the score on the mobility scale as the dependent variable. Therefore, the mobility scale was dichotomised into codes 0 
Table 1 Variables used in the analyses

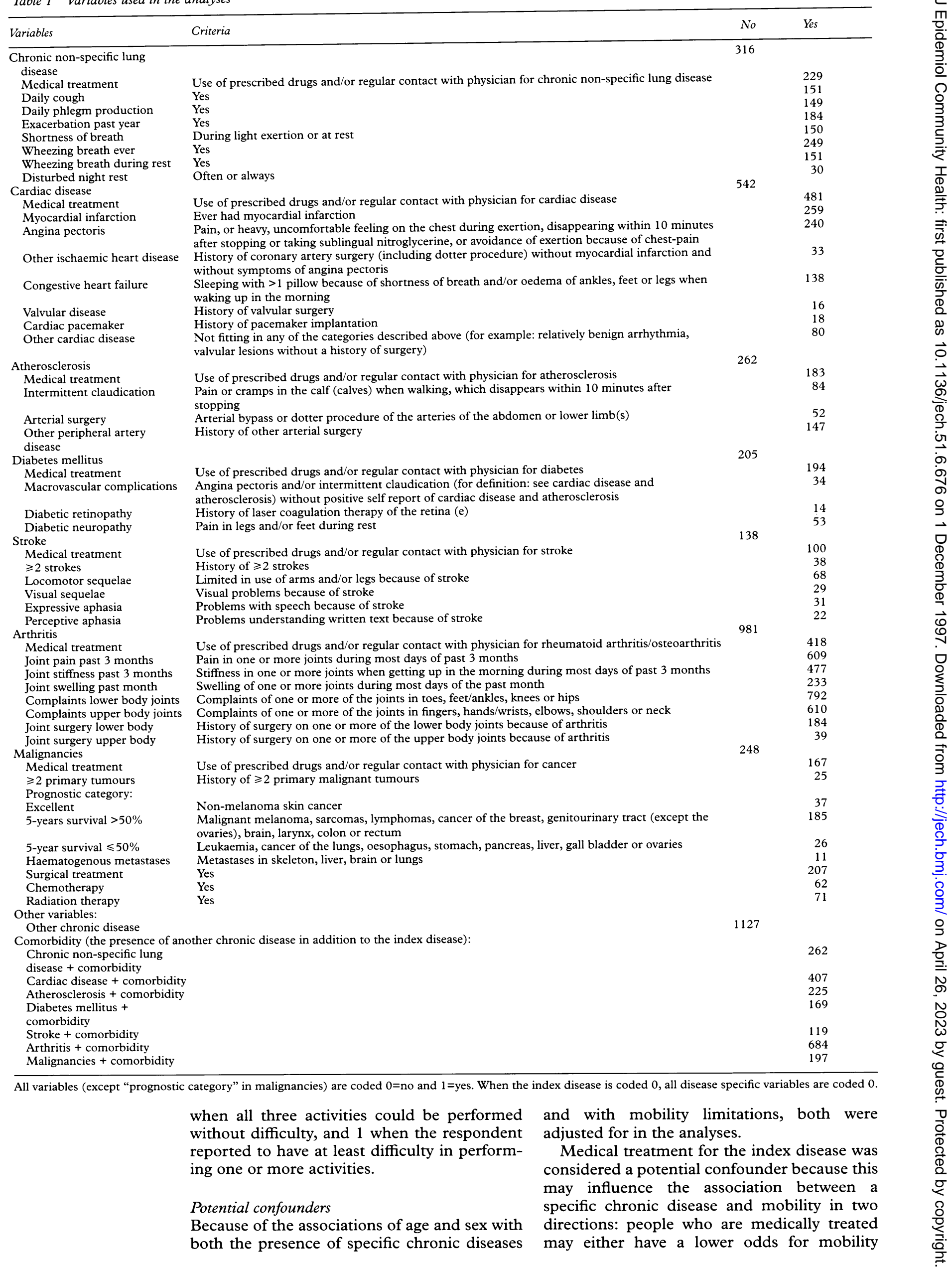


limitations because of this treatment, or have a higher odds when treatment has been started because of relatively severe disease. For all specific index diseases, continuous medical treatment was considered present when the patient reported to use prescribed drugs, or to have regular contact with a physician for the index disease, or both.

Finally, to answer the second research question, the presence of any other chronic disease in addition to the index disease (comorbidity) was adjusted for in the analyses as a potential confounder. Comorbidity was operationalised as a dichotomous variable and defined present when either one or more of the other explicitly asked diseases was present or the patient indicated to have any other chronic disease, or both.

\section{ANALYSES}

To answer the first research question, logistic regression analysis was performed using the total study sample. The analysis was adjusted for age and sex. To compare the strengths of the associations between specific chronic diseases and mobility limitations, all specific chronic diseases, including the category "other chronic disease", were entered in the model simultaneously.

To answer the second research question, logistic regression analyses were performed for each specific chronic disease separately. Subjects included in each model were those without any chronic disease (reference group) and those with at least the specific disease studied (the index disease). For example, in the logistic regression model examining the additional influence of parameters of clinical severity on the explanation of mobility limitations in patients with cardiac disease, subjects included were those who reported cardiac disease to be present and those who reported no chronic disease at all. Independent variables were included in the models in four steps.

In the first step, the potential confounders sex, age, and the presence of any other chronic disease in addition to the index disease (comorbidity) were entered. In the second step, the index disease was entered. In the third step, medical treatment for the index disease was entered because of its role as a potential confounder. Finally, in the fourth step the disease specific characteristics reflecting the clinical severity of the index disease were considered for stepwise entry. Variables included in this final step were entered into the model when a significant contribution to the explanation of mobility limitations was present $\left(\mathrm{p}_{\text {in }}<0.05\right)$. These parameters of clinical disease severity have an additional influence on mobility, conditional on self reported disease presence, when the final model is a significant improvement compared with the previous one that is adjusted for age, sex, comorbidity, and medical care for the index disease. This is judged by the change in $-2 \log$ likelihood and the significance of its improvement from the third to the fourth step.

The disease specific analyses may be sensitive to the problem of colinearity because of our decision to use the subjects without any chronic disease as a reference group for all disease specific models. As a result, colinearity between the independent variables may have been introduced because the variables concerning medical treatment, clinical disease severity, and comorbidity could be scored only for those subjects who reported the index disease (in the reference group, these variables are coded 0 ). To determine the extent to which colinearity was present, additional logistic regression analyses were performed for each of the specific chronic diseases separately, including only those subjects who reported the index disease. In these analyses, adjusted for the potential confounders age, sex, comorbidity, and medical treatment for the index disease, the disease specific characteristics reflecting clinical disease severity were considered for stepwise entry as described before (fourth step of the disease specific analyses). For each specific chronic disease, (the index diseases: chronic non-specific lung disease, cardiac disease, peripheral atherosclerosis, diabetes mellitus, stroke, arthritis, and malignancies) the strengths of the associations between the disease specific characteristics and mobility limitations among the subjects with the index disease were comparable to those resulting from the analyses described in the previous paragraph (in which the reference group of subjects without any chronic disease was included), as judged from the odds ratios and $95 \%$ confidence intervals. Thus, colinearity was shown not to be an important problem in the disease specific analyses.

It should be noted that subjects who reported more than one chronic disease are included in more than one regression model: for instance, a person with diabetes mellitus and chronic non-specific lung disease appears both in the model with diabetes as the index disease, and in that with chronic non-specific lung disease as the index disease.

\section{Results}

Table 2 shows the background characteristics of the study population. Table 3 shows the results pertaining to the first research question. Adjusted for age and sex, the presence of any of the specific chronic diseases is significantly associated with a higher odds for mobility limitations, with odds ratios (ORs) varying from 1.38 for malignancies to 3.37 for arthritis (see table 3). As was expected, stroke and arthritis show the largest ORs (3.29 and 3.37, respectively), followed by chronic non-specific lung disease and cardiac disease (2.34 and 2.11, respectively).

In table 4 , the results are shown pertaining to the second research question, concerning the additional explanation of mobility limitations by parameters of clinical disease severity. The results of the disease specific logistic regression analyses are presented for each step in the analysis for each disease separately.

In subjects with chronic non-specific lung disease, after adjustment for the potential confounders age, sex, and comorbidity, the presence of chronic non-specific lung disease 
Table 2 Basic characteristics of the study population $(n=2830)$

\begin{tabular}{|c|c|c|}
\hline Determinants & Number & $\%$ \\
\hline \multicolumn{3}{|l|}{ Sex } \\
\hline Male & 1379 & 48.7 \\
\hline Female & 1451 & 51.3 \\
\hline \multicolumn{3}{|l|}{ Age $(y)$} \\
\hline $55-64$ & 904 & 31.9 \\
\hline $65-74$ & 909 & 32.2 \\
\hline$\geqslant 75$ & 1017 & 35.9 \\
\hline \multicolumn{3}{|l|}{ Chronic diseases } \\
\hline Chronic non-specific lung disease & 316 & 11.2 \\
\hline Cardiac disease & 542 & 19.2 \\
\hline Peripheral atherosclerosis & 262 & 9.3 \\
\hline Diabetes mellitus & 205 & 7.2 \\
\hline Stroke & 138 & 4.9 \\
\hline Arthritis & 981 & 34.7 \\
\hline Malignancies & 248 & 8.8 \\
\hline Other chronic diseases & 1127 & 39.8 \\
\hline 0 Chronic diseases & 724 & 25.6 \\
\hline 1 Chronic disease & 960 & 33.9 \\
\hline$\geqslant 2$ Chronic diseases & 1146 & 40.5 \\
\hline \multicolumn{3}{|l|}{ Mobility limitations } \\
\hline \multicolumn{3}{|l|}{ Walking up and down 15 steps } \\
\hline Without difficulty & 2140 & 76.0 \\
\hline With difficulty & 412 & 14.6 \\
\hline Only with help/not at all & 265 & 9.4 \\
\hline \multicolumn{3}{|l|}{ Using own/public transportation } \\
\hline Without difficulty & 2470 & 87.4 \\
\hline With difficulty & 150 & 5.3 \\
\hline Only with help/not at all & 206 & 7.3 \\
\hline \multicolumn{3}{|l|}{ Cutting toenails } \\
\hline Without difficulty & 2018 & 71.6 \\
\hline With difficulty & 374 & 13.2 \\
\hline Only with help/not at all & 428 & 15.2 \\
\hline \multicolumn{3}{|l|}{ Difficulty with $\geqslant 1$ activity } \\
\hline No & 1733 & 61.8 \\
\hline Yes & 1072 & 38.2 \\
\hline
\end{tabular}

Table 3 Results of multiple logistic regression analyses (odds ratios and $95 \%$ confidence intervals) of age, sex, and specific chronic diseases on mobility limitations in the total study sample $\left(n_{t o t}=2805\right)$

\begin{tabular}{lcrl}
\hline & $\begin{array}{l}\text { Number } \\
\text { who } \\
\text { replied yes }\end{array}$ & $\begin{array}{l}\text { Odds } \\
\text { ratios }\end{array}$ & $\begin{array}{l}\text { 95\% } \\
\text { Confidence } \\
\text { intervals }\end{array}$ \\
\hline Age (per year) & & 1.10 & $1.09,1.11$ \\
Sex (female versus male) & & 1.76 & $1.45,2.13$ \\
Chronic diseases (yes versus no) & & \\
$\quad$ Chronic non-specific & 316 & 2.34 & $1.76,3.10$ \\
$\quad$ lung disease & 542 & 2.11 & $1.67,2.68$ \\
Cardiac disease & 262 & 1.56 & $1.14,1.97$ \\
Peripheral atherosclerosis & 205 & 1.89 & $1.33,2.68$ \\
Diabetes mellitus & 138 & 3.29 & $2.10,5.17$ \\
Stroke & 981 & 3.37 & $2.78,4.07$ \\
Arthritis & 248 & 1.38 & $1.02,1.88$ \\
Malignancies & 1127 & 1.92 & $1.60,2.31$ \\
$\quad$ Other chronic disease & & 2824.95 & \\
-2 log likelihood & & 75.0 & \\
\% predicted correctly & & & \\
\hline
\end{tabular}

itself does not add significantly to the explanation of mobility limitations, compared with subjects without any chronic disease (step 2: OR $1.49 ; 95 \%$ CI $0.70,3.16$ ). However, in patients with chronic non-specific lung disease, medical treatment for the disease is an explanatory factor with regard to the presence of mobility limitations (step 3: OR 4.44; 95\% CI 2.40, 8.21). In addition to medical treatment for chronic non-specific lung disease, a positive self report of shortness of breath during light exertion or at rest (step 4: OR $3.24 ; 95 \%$ CI $1.79,5.86)$ and regular disturbance of night rest resulting from chronic non-specific lung disease (step 4: OR 3.65; $95 \%$ CI 1.12, 11.83) are associated with the presence of mobility limitations. Thus, from the model resulting after the final step, it seems that the presence of mobility limitations in chronic non-specific lung disease patients is explained by shortness of breath and disturbed night rest as parameters of clinical severity of the disease, as well as by medical treatment for the disease.

A self report of cardiac disease is an explanatory factor for mobility limitations in these patients after adjustment for age, sex, and comorbidity (step 2: OR $2.56 ; 95 \%$ CI 1.58 , 4.15). However, the presence of cardiac disease does not explain mobility limitations when medical treatment is adjusted for (step 3: OR $1.03 ; 95 \%$ CI $0.47,2.29$ ). Medical treatment for cardiac disease is an explanatory factor for mobility limitations in cardiac patients (step 3: OR $2.69 ; 95 \%$ CI $1.37,5.31$ ). Conditional on the self reported presence of cardiac disease, symptoms of angina pectoris (step 4: OR 2.03; $95 \%$ CI $1.33,3.11$ ) or congestive heart failure (step 4: OR $1.98 ; 95 \%$ CI $1.19,3.30$ ) add to the explanation of mobility limitations in patients with cardiac disease. In the final model, adjusted for angina pectoris and congestive heart failure, medical treatment for cardiac disease (either use of medication or regular physician contacts), still adds to the explanation of mobility limitations, irrespective of the other variables in the model.

Adjusted for the potential confounders age, sex, and comorbidity, peripheral atherosclerosis does not add significantly to the explanation of mobility limitations (step 2: OR $0.66 ; 95 \%$ CI $0.22,2.01$ ). Also, in patients with peripheral atherosclerosis, medical treatment and disease specific characteristics do not add to the explanation of mobility limitations.

Compared with subjects without any chronic disease and adjusted for age, sex, and comorbidity, the self reported presence of diabetes is an explanatory factor for mobility limitations (step 2: OR 2.53; 95\% CI 1.14, 5.62). Neither medical treatment nor disease specific characteristics of diabetes (diabetic complications) have any additional influence on the explanation of mobility limitations. However, the net explanatory power of diabetes mellitus on mobility limitations loses its significance, when medical treatment is included in the regression model (step 3: OR 2.21; 95\% CI 0.43, 11.33). In this case, colinearity between a self report of diabetes and medical treatment is probably the cause, because $95 \%$ of the subjects reporting diabetes also reports to be medically treated for the disease.

Adjusted for age, sex, and comorbidity, a history of stroke explains the presence of mobility limitations (step 2: OR 4.73; 95\% CI $1.59,14.05)$. This is particularly so for patients who report to be medically treated for their stroke (step 3: OR 2.96; 95\% CI 1.13, 7.78). Certain disease specific characteristics, apart from medical treatment, significantly explain the association between stroke and mobility limitations. This applies to whether more than one stroke occurred (step 4: OR 4.03; 95\% CI $1.25,13.04)$, and the presence of visual sequelae (step 4: OR 5.45; 95\% CI 1.21, 24.59). The presence of locomotor sequelae did not add significantly to the explanation of mobility limitations in stroke patients. Although this 
Table 4 Results of multiple logistic regression analyses of mobility limitations (odds ratios and 95\% confidence intervals) on age, sex, comorbidity and disease specific characteristics in subjects with a specific chronic disease, compared with subjects without any chronic disease

\begin{tabular}{|c|c|c|c|c|c|c|c|c|c|}
\hline & \multirow[b]{2}{*}{$n_{\text {yes }}$} & \multicolumn{2}{|c|}{ Step 1 - enter } & \multicolumn{2}{|c|}{ Step 2 - enter } & \multicolumn{2}{|c|}{ Step 3 - enter } & \multicolumn{2}{|c|}{ Step 4 - stepwise } \\
\hline & & OR & $95 \% C I$ & $O R$ & $95 \% C I$ & $O R$ & $95 \% C I$ & $O R$ & $95 \% C I$ \\
\hline \multicolumn{10}{|c|}{ Chronic non-specific lung disease $n_{\text {tot }}=1026$} \\
\hline Sex (female versus male) & & 1.72 & $1.21,2.45$ & 1.75 & $1.22,2.49$ & 1.76 & $1.23,2.52$ & 1.72 & $1.19,2.47$ \\
\hline Comorbidity (yes versus no) & 262 & 12.21 & $8.47,17.59$ & 8.47 & $3.90,18.43$ & 10.68 & $4.76,24.01$ & 9.84 & $4.22,22.95$ \\
\hline Chronic non-specific lung disease & 316 & & & 1.49 & $0.70,3.16$ & 0.42 & $0.16,1.09$ & 0.31 & $0.11,0.86$ \\
\hline Medical treatment & 229 & & & & & 4.44 & $2.40,8.21$ & 3.03 & $1.58,5.83$ \\
\hline Shortness of breath & 150 & & & & & & & 3.24 & $1.79,5.86$ \\
\hline Disturbed night rest & 30 & & & & & & & 3.65 & $1.12,11.83$ \\
\hline $\begin{array}{l}-2 \text { log likelihood ( } \mathrm{p} \text { value } \\
\text { improvement) }\end{array}$ & & 818.07 & & 817.05 & (NS) & 793.73 & $(<0.0001)$ & 771.63 & $(<0.0001)$ \\
\hline$\%$ predicted correctly & & 82.4 & & 82.3 & & 83.0 & & 84.5 & \\
\hline \multicolumn{10}{|l|}{ Cardiac disease $n_{t o t}=1243$} \\
\hline Age (per year) & & 1.11 & $1.09,1.13$ & 1.11 & $1.09,1.13$ & 1.11 & $1.09,1.13$ & 1.11 & $1.09,1.13$ \\
\hline Sex (female versus male) & & 1.78 & $1.32,2.41$ & 1.97 & $1.44,2.70$ & 2.00 & $1.46,2.74$ & 1.86 & $1.35,2.56$ \\
\hline Comorbidity (yes versus no) & 407 & 8.12 & $6.01,10.97$ & 3.81 & $2.37,6.15$ & 3.92 & $2.43,6.34$ & 3.20 & $1.95,5.26$ \\
\hline Cardiac disease & 542 & & & 2.56 & $1.58,4.15$ & 1.03 & $0.47,2.29$ & 0.84 & $0.37,1.91$ \\
\hline Medical treatment & 481 & & & & & 2.69 & $1.37,5.31$ & 2.38 & $1.18,4.80$ \\
\hline Angina pectoris & 240 & & & & & & & 2.03 & $1.33,3.11$ \\
\hline Congestive heart failure & 138 & & & & & & & 1.98 & $1.19,3.30$ \\
\hline $\begin{array}{l}-2 \text { log likelihood ( } \mathrm{p} \text { value } \\
\text { improvement) }\end{array}$ & & 1091.00 & & 1077.30 & $(<0.001)$ & 1068.74 & $(<0.01)$ & 1047.64 & $(<0.001)$ \\
\hline$\%$ predicted correctly & & 80.3 & & 80.1 & $(20.00 \pi)$ & 80.2 & $(-0.01)$ & 80.6 & $(-0.001)$ \\
\hline \multicolumn{10}{|l|}{ Atherosclerosis $n_{t o t}=980$} \\
\hline Age (per year) & & 1.13 & $1.11,1.16$ & 1.13 & $1.11,1.16$ & 1.13 & $1.11,1.16$ & 1.13 & $1.11,1.16$ \\
\hline Sex (female versus male) & & 2.09 & $1.44,3.03$ & 2.08 & $1.44,3.02$ & 2.14 & $1.47,3.11$ & 2.14 & $1.47,3.11$ \\
\hline Comorbidity (yes versus no) & 225 & 11.18 & $7.58,16.48$ & 16.66 & $5.33,52.06$ & 17.09 & $5.43,53.80$ & 17.09 & $5.43,53.80$ \\
\hline Atherosclerosis & 262 & & & 0.66 & $0.22,2.01$ & 0.46 & $0.14,1.58$ & 0.46 & $0.14,1.58$ \\
\hline Medical treatment & 183 & & & & & 1.61 & $0.84,3.09$ & 1.61 & $0.84,3.09$ \\
\hline $\begin{array}{l}-2 \text { log likelihood ( } p \text { value } \\
\text { improvement) }\end{array}$ & & 741.17 & & 740.59 & (NS) & 738.53 & (NS) & 738.53 & (NS) \\
\hline$\%$ predicted correctly & & 83.4 & & 83.4 & & 83.8 & & 83.8 & \\
\hline \multicolumn{10}{|l|}{ Diabetes mellitus $n_{\text {tot }}=922$} \\
\hline Age (per year) & & 1.14 & $1.11,1.17$ & 1.14 & $1.11,1.17$ & 1.14 & $1.11,1.17$ & 1.14 & $1.11,1.17$ \\
\hline Sex (female versus male) & & 2.36 & $1.60,3.47$ & 2.37 & $1.61,3.50$ & 2.38 & $1.61,3.50$ & 2.38 & $1.61,3.50$ \\
\hline Comorbidity (yes versus no) & 169 & 9.44 & $6.16,14.47$ & 3.99 & $1.71,9.30$ & 3.98 & $1.70,9.28$ & 3.98 & $1.70,9.28$ \\
\hline Diabetes mellitus & 205 & & & 2.53 & $1.14,5.62$ & 2.21 & $0.43,11.33$ & 2.21 & $0.43,11.33$ \\
\hline Medical treatment & 194 & & & & & 1.16 & $0.25,5.29$ & 1.16 & $0.25,5.29$ \\
\hline $\begin{array}{l}-2 \text { log likelihood ( } \mathrm{p} \text { value } \\
\text { improvement) }\end{array}$ & & 694.15 & & 689.31 & $(<0.05)$ & 689.27 & (NS) & 689.27 & (NS) \\
\hline$\%$ predicted correctly & & 83.4 & & 83.6 & & 83.6 & & 83.6 & \\
\hline Stroke $n_{\text {tot }}=852$ & & & & & & & & & \\
\hline Age (per year) & & 1.14 & $1.11,1.17$ & 1.14 & $1.11,1.17$ & 1.14 & $1.11,1.17$ & 1.14 & $1.11,1.18$ \\
\hline Sex (female versus male) & & 1.91 & $1.26,2.90$ & 2.02 & $1.32,3.08$ & 2.00 & $1.31,3.06$ & 2.07 & $1.35,3.19$ \\
\hline Comorbidity (yes versus no) & 119 & 15.77 & $9.21,26.99$ & 3.56 & $1.11,11.40$ & 4.46 & $1.36,14.64$ & 3.11 & $0.88,10.93$ \\
\hline Stroke & 138 & & & 4.73 & $1.59,14.05$ & 1.86 & $0.47,7.29$ & 1.53 & $0.37,6.37$ \\
\hline Medical treatment & 100 & & & & & 2.96 & $1.13,7.78$ & 2.58 & $0.97,6.84$ \\
\hline$\geqslant 1$ stroke & 38 & & & & & & & 4.03 & $1.25,13.04$ \\
\hline Visual sequelae & 29 & & & & & & & 5.45 & $1.21,24.59$ \\
\hline$-2 \log$ likelihood ( $p$ value & & & & & & & & & \\
\hline improvement) & & 609.20 & & 602.15 & $(<0.01)$ & 597.36 & $(<0.05)$ & 585.10 & $(<0.01)$ \\
\hline$\%$ predicted correctly & & 85.5 & & 85.5 & & 85.5 & & 85.7 & \\
\hline Arthritis $n_{t o t}=1681$ & & & & & & & & & \\
\hline Age (per year) & & 1.12 & $1.10,1.13$ & 1.12 & $1.10,1.14$ & 1.12 & $1.10,1.14$ & 1.12 & $1.11,1.14$ \\
\hline Sex (female versus male) & & 1.80 & $1.41,2.30$ & 1.52 & $1.18,1.96$ & 1.46 & $1.13,1.88$ & 1.38 & $1.06,1.79$ \\
\hline Comorbidity (yes versus no) & 684 & 5.89 & $4.63,7.49$ & 2.33 & $1.71,3.18$ & 2.28 & $1.67,3.11$ & 2.24 & $1.63,3.08$ \\
\hline Arthritis & 981 & & & 4.62 & $3.26,6.55$ & 3.73 & $2.58,5.39$ & 1.51 & $0.93,2.45$ \\
\hline Medical treatment & 418 & & & & & 1.78 & $1.32,2.40$ & 1.40 & $1.03,1.92$ \\
\hline Joint stiffness & 477 & & & & & & & 1.87 & $1.37,2.54$ \\
\hline Complaints lower body & 792 & & & & & & & 2.28 & $1.55,3.34$ \\
\hline Surgery lower body & 184 & & & & & & & 1.54 & $1.02,2.31$ \\
\hline$-2 \log$ likelihood ( $\mathrm{p}$ value & & & & & & & & & \\
\hline improvement) & & 1660.63 & & 1584.61 & $(<0.0001)$ & 1569.95 & $(<0.001)$ & 1525.79 & $(<0.0001)$ \\
\hline$\%$ predicted correctly & & 75.9 & & 76.9 & & 77.5 & & 78.8 & \\
\hline Malignancies $n_{t o t}=959$ & & & & & & & & & \\
\hline Age (per year) & & 1.13 & $1.10,1.15$ & 1.13 & $1.10,1.15$ & 1.13 & $1.10,1.15$ & 1.13 & $1.10,1.15$ \\
\hline Sex (female versus male) & & 1.53 & $1.05,2.21$ & 1.51 & $1.04,2.19$ & 1.49 & $1.03,2.17$ & 1.55 & $1.06,2.25$ \\
\hline Comorbidity (yes versus no) & 197 & 7.69 & $5.21,11.35$ & 6.00 & $2.60,13.81$ & 5.89 & $2.55,13.60$ & 5.90 & $2.55,13.62$ \\
\hline Malignancies & 248 & & & 1.31 & $0.58,2.94$ & 1.52 & $0.60,3.85$ & 1.51 & $0.59,3.83$ \\
\hline Medical treatment & 167 & & & & & 0.82 & $0.44,1.53$ & 0.75 & $0.40,1.41$ \\
\hline Haematogenous metastases & 11 & & & & & & & 4.99 & $0.98,25.54$ \\
\hline$-2 \log$ likelihood ( $\mathrm{p}$ value & & & & & & & & & \\
\hline improvement) & & 759.07 & & 758.66 & (NS) & 758.28 & (NS) & 754.10 & $(<0.05)$ \\
\hline$\%$ predicted correctly & & 82.1 & & 82.1 & & 81.8 & & 82.1 & \\
\hline
\end{tabular}

might have been caused by colinearity the lack of an association between the occurrence of more than one stroke and locomotor sequelae renders this explanation unlikely.

The association between arthritis and mobility limitations (step 2: OR $4.62 ; 95 \%$ CI 3.26 , 6.55 ) is largely explained by medical treatment and the presence of disease specific characteristics reflecting clinical severity (step 4: OR $1.51 ; 95 \%$ CI $0.93,2.45)$. In addition to the presence of medical treatment (step 4: OR $1.40 ; 95 \%$ CI $1.03,1.92$ ), disease specific variables that are associated with mobility limitations in arthritis patients are joint stiffness (step 
4: OR 1.87; 95\% CI 1.37, 2.54), complaints of the joints in the lower body (step 4: OR 2.28; $95 \%$ CI $1.55,3.34$ ), and a history of joint surgery of the joints in the lower body, such as total hip replacement (step 4: OR $1.54 ; 95 \%$ CI $1.02,2.31$ ).

Adjusted for age, sex, and comorbidity, a history of cancer is not of influence on the presence of mobility limitations (step 2: OR $1.31 ; 95 \%$ CI $0.58,2.94)$. Moreover, neither medical treatment nor disease specific characteristics add to the explanation of mobility limitations in subjects with a history of cancer, compared with those without any chronic disease. The only disease specific characteristic that was of borderline significance in explaining mobility limitations among cancer patients, was the presence of haematogenous metastases (step 4: OR 4.99; 95\% CI 0.98, 25.54), but the number of patients with metastases is small $(n=11)$. We also performed the analyses with exclusion of those subjects with a history of non-melanoma skin cancer, but these results did not differ from those including all cancer patients.

\section{Discussion}

In the total study sample, each self reported chronic disease is significantly associated with the presence of mobility limitations. As expected, the strongest associations are found for chronic diseases of which the clinical picture includes disability (stroke and arthritis), or that are generally associated with decreased endurance capacity (chronic non-specific lung disease and cardiac disease). This finding is consistent with results from previous studies. $^{26-9}$

This study, however, shows that the self reported presence of specific chronic diseases is too crude a measure to be sufficiently accurate in explaining the presence of mobility limitations. After adjustment for the potential confounders age, sex, and comorbidity, the associations between the specific chronic diseases and mobility limitations seem to be largely explained by disease specific characteristics (including medical treatment for the index disease) reflecting clinical severity of the disease.

Symptoms explaning mobility limitations in case of a positive self report of chronic non-specific lung disease (shortness of breath during light exertion, and regular disturbance of night rest) are associated with a continuously decreased endurance tolerance. This is not the case for the other symptoms of chronic non-specific lung disease (for example, coughing and wheezing), which are, at the most, only limiting during an episode. In addition, comorbidity and medical treatment for chronic nonspecific lung disease were shown to be explanatory factors for mobility limitations in patients with chronic non-specific lung disease. Thus, the association of chronic non-specific lung disease with mobility limitations can be explained by parameters of clinical disease severity (shortness of breath and disturbed night rest, as well as medical treatment) and the presence of other chronic diseases in addition to the chronic non-specific lung disease.

For cardiac disease, symptoms of angina pectoris and congestive heart failure, both associated with decreased endurance capacity, added significantly to the explanation of mobility limitations, whereas the other characteristics studied (such as a history of myocardial infarction or cardiac surgery) did not. The association between the self reported presence of cardiac disease and mobility limitations seems to be explained completely by medical treatment and specific cardiac diagnoses indicative of the clinical severity (angina pectoris and congestive heart failure), as well as by the presence of comorbidity.

For atherosclerosis, disease specific characteristics did not add to the explanation of mobility limitations. For peripheral atherosclerosis, it has been shown that the accuracy of patients' self reports, compared with general practitioners' information, is low. ${ }^{10}$ Therefore, it might be expected that symptoms would add to the explanation of mobility limitations in this chronic disease, but this was not the case. Relevant bias can be suspected because of confusion between the Dutch words "slagaderen" (arteries) and "spataderen" (varicose veins, venous insufficiency). Venous insufficiency may also be associated with mobility limitations, but symptoms are different from those of peripheral atherosclerosis. The explanatory power of comorbidity on mobility limitations in patients reporting peripheral atherosclerosis is high, compared with that in patients with other index diseases.

In patients with diabetes mellitus, disease specific characteristics reflecting clinical severity (that is, the presence of microvascular and macrovascular complications) did not add significantly to the explanation of mobility limitations. The possibility that these disease specific characteristics, particularly macrovascular complications, could be shown to influence the explanation of mobility limitations in diabetes mellitus, was diminished by the decision not to consider the additional presence of a self report of cardiac disease or atherosclerosis as manifestations of macrovascular complications, but to include these as comorbidity. The prevalence of cardiac disease and peripheral atherosclerosis among patients with diabetes mellitus was high, compared with the prevalence in the total sample $(28.8 \%$ versus $19.2 \%$ for cardiac disease, $19.0 \%$ versus $9.3 \%$ for atherosclerosis), and compared with the prevalence of macrovascular complications in diabetic patients $(16.6 \%)$. In contrast with the disease specific complications, the presence of comorbidity adds to the explanation of mobility limitations in patients with diabetes mellitus. The fact that virtually all diabetic patients are medically treated for the disease, which introduces the problem of colinearity in the analysis, probably explains why the association of diabetes mellitus with mobility limitations is not significant anymore, once medical treatment is entered as a potential confounder.

In contrast with expectations, the presence of locomotor sequelae in stroke patients did 
not add to the explanation of mobility limitations. Although the prevalence of this symptom is high $(49.3 \%)$, a history of more than one stroke and the presence of visual sequelae, which were both not associated with the presence of locomotor sequelae, seem to be more important. These aspects of clinical severity largely explain the association between stroke and mobility limitations. In contrast with the results in the other index diseases, comorbidity offers no additional explanation.

In patients with arthritis, affliction of the lower body joints has the largest explanatory power on mobility limitations, as would be expected considering the content of the mobility items included. Also, a history of lower body joint surgery (an intervention with the purpose of restoring function and alleviating symptoms) adds to the explanation of mobility limitations. The conclusion that can be drawn from this cross sectional analysis is that, although joint surgery undoubtedly has a beneficial influence on functioning and symptoms, mobility is not restored fully, compared with people of the same age and sex, but without chronic diseases. The association between arthritis and mobility limitations is thus explained by certain parameters of disease severity (joint stiffness, complaints of lower body joints, and a history of surgery on the lower body joints), as well as by continuous medical treatment and the presence of comorbidity.

The cross sectional design of this study is a possible explanation for the negative results we found for patients with a history of cancer: in all prognostic categories, only survivors are included (the mean duration of time since diagnosis was approximately eight years). In the absence of haematogenous metastases and additional chronic diseases, the initial severity of cancer (as reflected by the prognostic category) does not add to the explanation of mobility limitations.

Medical treatment was adjusted for, because it was expected that the associations between the specific index diseases and mobility could be modified by treatment in both directions. However, when medical treatment was of influence (in chronic non-specific lung disease, cardiac disease, and arthritis), it was consistently associated with a higher odds for mobility limitations. A possible explanation is that only patients with comparativley severe chronic non-specific lung disease, cardiac disease or arthritis will receive continuous medical treatment (medication or regular check ups by a physician). Although their symptoms may be alleviated to a great extent, they may still experience mobility limitations more often than people with less severe disease. For those diseases for which continuous medical treatment is the rule, such as diabetes mellitus, stroke or cancer, inclusion of medical treatment in the model will not add significantly to the explanation of mobility limitations because of its low variance among patients.

As described in the methods section, our study population is a comparatively healthy selection of the original sample, which may have caused an underestimation of the observed associations.

Summarising, this study provides evidence that a more accurate explanation of the associations between specific chronic diseases and mobility limitations can be obtained by including questions on medical treatment and disease specific signs and symptoms reflecting clinical disease severity, conditional on a positive self report of the index disease. This method of acquiring additional information reflecting the clinical severity of chronic diseases provides a cost saving alternative for other methods that are frequently used, such as medical record extraction, ${ }^{20-22}$ laboratory tests, ${ }^{23}$ or clinical examination. ${ }^{24}$ For large scale population surveys, the organisational and financial demands associated with the use of these methods will often exceed the available resources. Moreover, there may be some doubt as to whether these measures, which are often developed in specific clinical settings, are equally applicable in the general population. Our results also provide evidence supporting the usefulness of distinguishing between specific chronic diseases when studying the impact of disease on aspects of physical functioning, such as the presence of mobility limitations, instead of using measures in which the information about the specific chronic diseases is pooled into one overall score, as is the case for many other measures of disease severity. ${ }^{22} 25$ Use of pooled information excludes the possibility to investigate the impact of a specific chronic disease on the outcome that is being studied, be it mobility, mortality or the use of health care facilities.

In view of our results, future predictions regarding the prevalence of mobility limitations may have to be adjusted, but additional investigations are needed to determine to what extent. Currently, predictions are mostly computed using trends in the prevalences of self reported presence of diseases and mobility limitations. The most important gain of this study, compared with previous ones, is that it is shown that the presence of disease specific characteristics reflecting clinical severity in patients with a specific chronic disease, rather than only the positive self report of a disease, explains the presence of mobility limitations. The use of this type of additional information, reflecting the clinical severity of a disease, may increase the accuracy of future predictions regarding the development of the prevalence of mobility limitations or disability. Moreover, differences between studies on the associations between chronic diseases and physical functioning may be explained by differences in the prevalences of disease specific signs and symptoms in diagnosed and treated patients. For instance, the conflicting evidence with regard to future developments of disability free life expectancy as far as "compression" or "decompression" of morbidity is concerned ${ }^{29-34}$ may partly result from a different distribution of clinical disease severity (in other words, different prevalences of signs and symptoms) across countries. Differences in this distribution may, for example, reflect differences in the extent to 
which diseases are diagnosed in early stages (a large proportion of diseases diagnosed by screening may result in relatively more people with less severe disease). From a longitudinal perspective, using information on both the presence of chronic diseases and on disease specific characteristics reflecting clinical disease severity, makes it possible for future predictions to take into account the effects of changes in the distribution of clinical severity of specific chronic diseases. The occurrence of these type of changes is likely to occur. For example, increases in the early detection of chronic diseases and improvements in medical treatment for these early stages, including treatment of risk factors for other diseases (such as treatment of hypertension to prevent cardiovascular and cerebrovascular disease) may lead to a shift of the distribution of clinical severity towards less severe disease, and to a decrease in the associations of specific chronic diseases with disability when the changes in clinical severity are not taken into account.

This study is based on data collected in the context of the Longitudinal Aging Study Amsterdam (LASA), conducted at the Department of Psychiatry and the Department of Sociology and Social Gerontology of the Vrije Universiteit, Amsterdam, the Netherlands.

Funding: LASA is largely funded by the Ministry of Health, Welfare and Sports of the Dutch Government. Conflicts of interest: none.

1 Cornoni-Huntley JC, Foley DJ, Guralnik JM. Co-morbidity analysis: a strategy for understanding mortality, disability and use of health care facility of older people. Int $\mathscr{f}$ Epidemiol 1991;20 (suppl1):S8-17.

2 Verbrugge LM, Lepkowski JM, Imanaka Y. Comorbidity and its impact on disability. Milbank $Q$ 1989;67:450-84.

3 Guralnik JM, LaCroix AZ, Everett DF. Comorbidity of chronic conditions and disability among older persons United States, 1984. F Am Med Assoc 1990;263:209-10.

4 Guralnik JM, LaCroix AZ, Abbott RD, et al. Maintaining mobility in late life. I. Demographic characteristics and chronic conditions. Am f Epidemiol 1993;137:845-57.

5 Verbrugge LM, Lepkowski JM, Konkol LL. Levels of Verbrugge LM, Lepkowski JM, Konkol LL. Levels of
disability among US adults with arthritis. f Gerontol 1991; disability am.

6 Boult C, Kane RL, Louis TA, Boult L, McCaffrey D. Chronic conditions that lead to functional limitation in the elderly. F Gerontol 1994;49:M28-36.

7 Guccione AA, Felson DT, Anderson JJ, et al. The effects of specific medical conditions on the functional limitations of elders in the Framingham study. Am $f$ Publ Health 1994;84:351-8.

8 Bos GAM van den. The burden of chronic diseases in terms of disability, use of health care and healthy life expectancies. Eur $\mathcal{F}$ Publ Health 1995;5:29-34.

9 Verbrugge LM. New thinking and science on disability in mid- and late life. Eur $\mathcal{F}$ Publ Health 1995;5:20-8.

10 Kriegsman DMW, Penninx BWJH, Eijk JThM van, Boeke AJP, Deeg DJH. Self-reports and general practitioner information on the presence of chronic diseases in community dwelling elderly: a study on the accuracy of patients' self-reports and on determinants of inaccuracy. $f$ Clin Epiself-reports and on determin
demiol $1996 ; 49: 1407-17$.

11 Harlow SD, Linet MS. Agreement between questionnaire data and medical records. The evidence for accuracy of recall. Am f Epidemiol 1989;129:233-48.

12 Bos GAM van den. Care for the chronically ill. (In Dutch: Zorgen van en voor chronisch zieken). [PhD thesis] Amsterdam: Bohn, Scheltema and Holkema, 1989.

13 Kehoe R, Wu S-Y, Leske MC, Chylack LT. Comparing selfreported and physician-reported medical history. $\mathrm{Am} f$ Epidemiol 1994;139:813-8.
14 Deeg DJH, Knipscheer CPM, Tilburg W van, eds. Autonomy and well-being in the aging population. Concepts and design of the Longitudinal Aging Study Amsterdam. Bunnik: Netherlands Institute for Gerontology, 1993.

15 Broese van Groenou MI, Tilburg TG van, Leeuw ED de, Liefbroer AC. Data collection. In: Knipscheer CPM, JongGierveld J de, Tilburg TG van, Dykstra PA, eds. Living arrangements and social networks of older adults. First results. arrangements and social networks of older adults. First

16 Smit JH, Vries MZ de. Procedures and results of the field work. In: Deeg DJH, Westendorp-de Serrière $M$, eds Autonomy and well-being in the aging population. I. Repor from the Longitudinal Aging Study Amsterdam 1992-1993. Amsterdam: VU University Press, 1994:7-13.

17 Rose GA. The diagnosis of ischaemic heart pain and intermittent claudication in field surveys. Bull World Health Org 1962;27:645-58.

18 Perez CA, Brady LW, eds. Principles and practice of radiation oncology. Philadelphia: Lippincott, 1992.

19 Deeg DJH, Kriegsman DMW, Zonneveld RJ van. Prevalence of four chronic conditions and their association with health limitations in older persons in The Netherlands, 1956-1993. (In Dutch: Prevalentie van vier chronische ziekten en hun samenhang met gezondheidsbeperkingen bij ouderen in Nederland, 1956-1993). Tijdschr Soc Gezondheidsz 1994;72:434-41.

20 Asenjo MA, Baré L, Bayas JM, et al. Relationship between severity, costs and claims of hospitalized patients using the Severity of Illness Index. Eur F Epidemiol 1994;10:625-32.

21 Hartz AJ, Guse C, Sigmann P, Krakauer H, Goldman RS Hagen TC. Severity of illness measures derived from the Hagen TC. Severity of illness measures derived from the
Uniform Clinical Data Set (UCDSS). Med Care 1994;32: Uniform

22 Parkerson GR, Broadhead WE, Tse CJ. The Duke Severity of Illness Checklist (DUSOI) for measurement of severity and comorbidity. $\mathcal{f}$ Clin Epidemiol 1993;46:379-93.

23 Damiano AM, Bergner M, Draper EA, Knaus WA, Wagner DP. Reliability of a measure of severity of illness: acute physiology of chronic health evaluation - II. $\mathcal{f}$ Clin Epidemiol 1992;45:93-101.

24 Kalra L, Dale P, Crome P. Evaluation of a clinical score for prognostic classification of elderly stroke patients. Age Ageing 1994;23:492-98.

25 Charlson ME, Pompei P, Ales KL, MacKenzie CR. A new method of classifying prognostic comorbidity in longitudinal studies: development and validation. $\mathcal{F}$ Chron Dis 1987 ; 40:373-83.

26 Charlson M, Szatrowski TP, Peterson J, Gold J. Validation of a combined comorbidity index. $\mathcal{F}$ Clin Epidemiol 1994;47: 1245-51

27 Parmelee PA, Thuras PD, Katz IR, Lawton MP. Validation of the Cumulative Illness Rating Scale in a geriatric residential population. f Am Geriatr Soc 1995;43:130-7.

28 Waite $K$, Oddone E, Weinberger M, Samsa G, Foy M Henderson W. Lack of association between patients' measured burden of disease and risk for hospital readmission. $f$ Clin Epidemiol 1994;47:1229-36.

29 Verbrugge LM. Recent, present and future health of American adults. Annu Rev Public Health 1989;10:333-61.

30 Olshansky SJ, Ault AB. The fourth stage of the epidemiologic transition: the age of delayed degenerative diseases. Milbank $Q$ 1986;64:355-91.

31 Manton KG, Corder LS, Stallard E. Estimates of change in chronic disability and institutional incidence and prevalence rates in the US elderly population from the 1982 , 1984, and 1989 National Long Term Care Survey. $\mathscr{f}$ Gerontol 1993;48:S153-66.

32 Roos NP, Havens B, Black C. Living longer but doing worse: assessing health status in elderly persons at two points in time in Manitoba, Canada, 1971 and 1983. Soc Sci Med 1993;36:273-82.

33 Corder LS. Improving survey measurement: health status transitions in a longitudinal list sample design. In: Mathers C, McCallum J, Robine J-M, eds. Advances in health expectancies: proceedings of the $7^{\text {h }}$ meeting of the International Network on Health Expectancy (REVES), Canberra, February 1994. Canberra: Australian Institute of Health and Welfare, 1994:408-23.

34 Verbrugge LM. The experience and measure of disability. In: Mathers C, McCallum J, Robine J-M, eds. Advances in health expectancies: proceedings of the $7^{h}$ meeting of the health expectancies: proceedings of the $7^{\text {h }}$ meeting of the
International Network on Health Expectancy (REVES), Canberra, February 1994. Canberra: Australian Institute of Health and Welfare, 1994:19-33. 